\title{
Conhecimento de cuidadores sobre a prevenção de lesões por pressão em crianças e adolescentes cadeirantes
}

\author{
Knowledge of caregivers about prevention of pressure injury in children and adolescents \\ wheelchair'users
}

Conocimiento de los cuidadores acerca de la prevención de lesiones por presión en niños y adolescentes de sillas de ruedas

Simone de Paula ${ }^{1 *}$, Eliane Fátima Manfio ${ }^{1}$, Kétlin Caroline Griebeler ${ }^{1}$, Maristela Cassia de Oliveira Peixoto ${ }^{1}$.

\section{RESUMO}

Objetivo: Avaliar o conhecimento de cuidadores sobre a prevenção de lesões por pressão em crianças e adolescentes cadeirantes. Métodos: Pesquisa transversal descritiva. Foram utilizadas duas entrevistas estruturadas contendo questões relacionadas ao conhecimento dos cuidadores sobre as estratégias de prevenção de lesões por pressão. Resultados: Participaram 11 cuidadoras, jovens, mães de crianças e adolescentes dependentes de cadeira de rodas com idade média de 33,3 $\pm 9,8$ anos. Um percentual de $90,9 \%$ das participantes não recebeu orientações de profissionais da saúde sobre medidas para prevenir lesões na pele. Além disso, $54,5 \%$ das participantes relataram que as crianças e adolescentes permaneciam mais de 13 horas diárias na posição sentada e referiram não realizarem manobras de alívio. A maioria das cuidadoras demonstrou um moderado nível de conhecimento sobre medidas preventivas. Conclusão: A pesquisa reforça a necessidade do desenvolvimento de programas educativos sobre medidas preventivas de lesões por pressão.

Palavras-chave: Lesão por pressão, Cuidadores, Cadeiras de rodas, Promoção da saúde.

\begin{abstract}
Objective: Analyze the knowledge of caregivers about the prevention of pressure injury in children and adolescents wheelchair' users. Methods: Transversal and descriptive research. Two structured interviews were performed with questions related to the knowledge of caregivers about strategies for the prevention of pressure injury. Results: The sample was composed of 11 caregivers of children and adolescents whose are wheelchair-dependent with mean age of $33.3 \pm 9.8$ years. The profile of caregivers consisted of young women with maternal relationship with child and fundamental level of schooling. A percentage of $90.9 \%$ of participants have not received orientation by health professional on strategies to prevent skin injuries. In addition, $54.5 \%$ of sample refers to spend more than 13 hours per day in sitting position and they do not perform relief maneuvers. Most caregivers demonstrated a moderate level of knowledge about strategies to prevent pressure injury. Conclusion: Research reinforces the need for the development of educational programs on strategies to prevent pressure injuries.
\end{abstract}

Key words: Pressure ulcer, Caregivers, Wheelchairs, Health promotion.

1 Universidade Feevale, Novo Hamburgo - RS. *E-mail: sdpaula@feevale.br

SUBMETIDO EM: 11/2019

ACEITO EM: 12/2019

PUBLICADO EM: 2/2020 


\section{RESUMEN}

Objetivo: Analisar el conocimiento de los cuidadores sobre la prevención de lesiones por presión en niños y adolescentes que usan sillas de ruedas. Métodos: Investigación transversal y descriptiva. Se realizaron dos entrevistas estructuradas con preguntas relacionadas con el conocimiento de los cuidadores sobre las estrategias para la prevención de lesiones por presión. Resultados: La muestra estuvo compuesta por 11 cuidadores de niños y adolescentes dependientes de sillas de ruedas con una edad promedio de $33.3 \pm 9.8$ años. El perfil de los cuidadores consistió en mujeres jóvenes con relación materna con el niño y nivel fundamental de escolaridad. Un porcentaje del $90.9 \%$ de los participantes no ha recibido orientaciónes por parte de lo profesional de la salud acerca de estrategias para prevenir lesiones cutáneas. Además, el 54.5\% de la muestra se refiere a pasar más de 13 horas por día en posición sentada y no realizan maniobras de alivio. La mayoría de los cuidadores demostraron un nivel moderado de conocimiento sobre estrategias para prevenir lesiones por presión. Conclusión: la investigación refuerza la necesidad de desarrollar programas educativos sobre estrategias para prevenir lesiones por presión.

Palabras clave: Úlcera por presión, Cuidadores, Silla de ruedas, Promoción de la salud.

\section{INTRODUÇÃO}

As lesões por pressão (LPP) constituem-se de uma área de dano na pele ou em estruturas subjacentes, normalmente sobre uma proeminência óssea, resultante da pressão, fricção e/ou suas combinações (NPUAP, 2014). Várias condições e fatores de risco contribuem para o desenvolvimento de LPP, incluindo a imobilidade) desnutrição, a percepção sensorial diminuída e a presença de incontinência urinária e fecal.

O preciso fator etiológico das LPP ainda permanece desconhecido, mas tem-se sugerido que, o prolongado tempo de isquemia tecidual resultante da compressão dos tecidos moles vascularizados é o principal mecanismo fisiopatológico para o início da morte celular e a irreversível necrose em áreas de risco para cadeirantes, tais como, a tuberosidade isquiática, o sacro e o cóccix (LUBOZ V, et al., 2014).

O perfil epidemiológico das LPP varia consideravelmente de acordo com a população estudada, mas estima-se que $5-20 \%$ dos indivíduos com alterações crônicas na mobilidade possam necessitar de cuidados relacionados ao aparecimento de feridas. Há poucos estudos de coorte sobre a incidência de LPP no followup de crianças e adolescentes com deficiências neuromotoras crônicas, mas sabe-se que há uma relação entre a severidade da deficiência, o tempo de permanência na posição sentada e o risco de desenvolvimento de LPP a longo prazo (ROLIM JA, et al., 2013).

Apesar dos avanços científicos e tecnológicos, as LPP ainda permanecem como um relevante problema de saúde pública, representando uma importante causa de morbidade e mortalidade em nível mundial. 0 desenvolvimento dessas lesões interfere na funcionalidade e na qualidade de vida do paciente e de seus cuidadores, podendo resultar em dor e infecções recorrentes.

Além disso, o difícil tratamento clínico das LPP pode aumentar e prolongar as internações hospitalares, constituindo-se numa expressiva sobrecarga econômica para os serviços de saúde (ROLIM JA, et al., 2013). Estudos recentes têm apontado que, apesar mecanismos fisiopatológicos envolvidos no aparecimento das LPP, a maioria das lesões cutâneas crônicas pode ser evitada. Em virtude da significância clínica e socioeconômica das LPP, as estratégias de prevenção ganham destaque na literatura científica, incluindo a utilização de programas de educação em saúde voltados aos cuidadores (PALFREYMAN SJ e STONE PW 2015).

O cuidador pode ser compreendido como aquela pessoa que presta cuidados à outra que necessite, por estar acamada, com limitações físicas ou mentais, com ou sem remuneração. Quando uma criança nasce com alterações neuromotoras, os cuidadores, especialmente os membros da família, utilizam o seu conhecimento sobre cuidado para criar um ambiente que atenda as demandas da criança (OLIVEIRA DK, et al., 2012). 
Neste contexto, o envolvimento e o conhecimento dos cuidadores sobre estratégias de prevenção de lesões por pressão em crianças e adolescentes cadeirantes são requisitos essenciais para o aumento da qualidade de vida e a redução de comorbidades em portadores de deficiências neuromotoras.

Frente às evidências apresentadas e a carência de estudos nesta área, a presente pesquisa apresenta o seguinte problema: qual o nível de informação das famílias de cadeirantes sobre cuidados preventivos? Com base nesta questão de pesquisa, o objetivo do presente estudo foi avaliar o conhecimento dos cuidadores sobre a prevenção de lesões por pressão em crianças e adolescentes usuários de cadeira de rodas.

\section{MÉTODOS}

O estudo caracterizou-se por uma pesquisa quantitativa do tipo transversal descritivo. O local de realização da pesquisa foi uma clínica-escola de Fisioterapia de uma universidade comunitária do Rio Grande do Sul. Os critérios de inclusão para a seleção da amostra foram: ser cuidador de criança ou adolescente com disfunção neuromotoras e dependente de cadeira de rodas; apresentar bom nível de compreensão; e comparecer às entrevistas no local e na data previamente estabelecidos.

Os cuidadores que atenderam a estes critérios foram contatados e convidados a participar da pesquisa mediante assinatura do Termo de Consentimento Livre e Esclarecido. Participaram deste estudo 11 cuidadores de crianças e adolescentes dependentes da cadeira de rodas com idade média de $33,3( \pm 9,8)$ anos que estavam em atendimento fisioterapêutico no período de março a outubro de 2017.

Para a coleta de dados foram utilizadas duas entrevistas estruturadas contento questões relacionadas ao perfil dos cuidados domiciliares e o nível de conhecimento dos cuidadores sobre as estratégias de prevenção de lesões por pressão. Em virtude da carência de instrumentos validados e específicos para a avaliação do conhecimento de cuidadores sobre a prevenção de lesões por pressão, as questões utilizadas nas entrevistas foram baseadas no estudo espanhol de Bellon JA (2014).

Este instrumento analisa itens relacionados à nutrição, hidratação, cuidados posturais e cuidados com a pele através de perguntas relacionadas a esta temática. Para responder aos questionamentos, os participantes da pesquisa responderam "verdadeiro" para confirmar a sentença, ou "falso" para negar a afirmativas segundo Bellon JA (2014).

Adicionalmente, dados clínicos e sociodemográficos também foram coletados durante a aplicação dos instrumentos. As entrevistas foram realizadas individualmente, através de um encontro em uma sala privativa, com tempo médio de 30 minutos para cada entrevista. Após a coleta e a tabulação, os dados foram analisados quantitativamente por meio de estatística descritiva e apresentados em percentuais, sob a forma de tabelas.

Este estudo foi aprovado pelo Comitê de Ética em Pesquisa da Universidade Feevale em 4 de março de 2016 (CAAE 52077315.8.0000.5348, parecer no 1.437.747). A pesquisa foi conduzida de acordo com os padrões éticos exigidos, seguindo as normas vigentes na Resolução № 466/12 do Conselho Nacional de Saúde, e suas complementares, que regulamentam a pesquisa envolvendo seres humanos.

\section{RESULTADOS}

Através da análise do perfil sociodemográfico dos participantes da pesquisa pode-se observar que a maioria dos cuidadores é do gênero feminino, com idade média de $33,3( \pm 9,8)$ anos, relação de parentesco materno com a criança e adolescente, e nível de escolaridade do ensino fundamental (Tabela 1). 
Tabela 1 - Perfil sociodemográfico do cuidador.

\begin{tabular}{lc}
\hline Idade do cuidador & \\
\hline Média (anos) & 33,3 \\
Desvio-padrão (anos) & $\pm 9,8$ \\
\hline Gênero (\%) & 90,9 \\
\hline Feminino & 9,09 \\
Masculino & 72,7 \\
\hline Relação de parentesco (\%) & 9,09 \\
\hline Mãe & 9,09 \\
Pai & 9,09 \\
Irmã & 63,6 \\
Avó & 36,3 \\
\hline Escolaridade (\%) & 0 \\
\hline Ensino fundamental & 54,5 \\
Ensino médio & 45,4 \\
Superior & \\
\hline Demais cuidadores (\%) & \\
\hline Sem auxílio & \\
Outros membros da família & \\
\hline
\end{tabular}

Fonte: De Paula S, et al., 2019.

Para o perfil clínico das crianças e adolescentes cadeirantes, observou-se que a maioria dos participantes é do gênero masculino, com média de 12 anos $( \pm 4,8)$, com diagnóstico predominante de doenças neuromusculares $(36,3 \%)$ e mielomeningocele $(27,2 \%)$ (Tabela 2$)$.

Tabela 2 - Perfil clínico das crianças e adolescentes cadeirantes.

\begin{tabular}{lc}
\hline Idade & \\
\hline Média (anos) & 12,0 \\
Desvio-padrão (anos) & $\pm 4,8$ \\
\hline Gênero (\%) & 45,4 \\
\hline Feminino & 54,5 \\
Masculino & \\
\hline Patologia (\%) & 36,3 \\
\hline Doenças neuromusculares & 18,1 \\
Paralisia cerebral & 27,2 \\
Mielomeningocele & 18,1 \\
Lesão medular traumática & \\
\hline
\end{tabular}

Fonte: De Paula S, et al., 2019. 
Em relação ao perfil do cuidado domiciliar, na Tabela 3, observa-se que $90,9 \%$ dos cuidadores não receberam orientações sobre a prevenção de LPP de profissionais da saúde. Em contrapartida, 81,8\% das crianças e adolescentes cadeirantes não apresentaram histórico de LPP. Em relação ao tempo de permanência na cadeira de rodas, $54,5 \%$ dos participantes permanecem mais de 13 horas na posição sentada.

Tabela 3 - Perfil do cuidado domiciliar relacionado à prevenção de LPP.

\begin{tabular}{|c|c|c|}
\hline & $\mathrm{n}$ & $\%$ \\
\hline \multicolumn{3}{|c|}{ Recebeu orientações para prevenção de LPP? } \\
\hline Sim & 1 & 9,0 \\
\hline Não & 10 & 90,9 \\
\hline \multicolumn{3}{|l|}{ Apresenta ou já apresentou LPP? } \\
\hline Sim & 2 & 18,1 \\
\hline Não & 9 & 81,8 \\
\hline \multicolumn{3}{|c|}{ Qual o tempo diário na cadeira de rodas? } \\
\hline$<6 \mathrm{~h}$ & 1 & 9,0 \\
\hline $7-12 \mathrm{~h}$ & 4 & 36,3 \\
\hline$>13 \mathrm{~h}$ & 6 & 54,5 \\
\hline \multicolumn{3}{|l|}{ Realiza manobras de alívio? } \\
\hline Sim & 5 & 45,4 \\
\hline Não & 6 & 54,5 \\
\hline \multicolumn{3}{|l|}{ Se sim, quais? } \\
\hline Inclina-se para os lados & 3 & 60,0 \\
\hline Empurra-se para cima & 0 & 0 \\
\hline Todos & 2 & 40,0 \\
\hline \multicolumn{3}{|l|}{ Por quanto tempo? } \\
\hline$<10$ segundos & 4 & 80,0 \\
\hline $10-30$ segundos & 1 & 20,0 \\
\hline \multicolumn{3}{|c|}{ Cuidados que realiza em casa para a prevenção de LPP? } \\
\hline Cremes hidratantes & 11 & 100 \\
\hline Almofadas especiais & 1 & 9,0 \\
\hline Almofadas com orifício & 7 & 63,6 \\
\hline Trocas frequentes de fralda & 3 & 27,2 \\
\hline Inspeção visual & 1 & 9,0 \\
\hline Ingestão de alimentos saudáveis & 3 & 27,2 \\
\hline Ingestão hídrica & 11 & 100 \\
\hline Evita traumas acidentais & 1 & 9,0 \\
\hline Adota cuidados com a postura & 11 & 100 \\
\hline
\end{tabular}

Fonte: De Paula S, et al., 2019.

Quando questionados sobre a realização de manobras de alívio, 54,5\% das crianças e adolescentes afirmaram não as realizar. Dos pacientes que realizam, a maioria refere realizar manobras de inclinação para os lados por menos de 10 segundos. Adicionalmente, $100 \%$ dos participantes declararam fazer uso de cremes hidratantes, ingerir 1 litro de água por dia e adotar cuidados com a postura. Com menor frequência, cuidados como a troca frequente de fraldas, a inspeção visual e a cautela com traumas acidentais também foram relatadas. 
Através da análise do nível de conhecimento dos cuidadores (Tabela 4) utilizando como base o estudo de Bellon JA (2014), todos os cuidadores afirmaram que tem consciência de que é necessário secar as zonas corporais predispostas a LPP, consumir 1 litro de água por dia e manter a postura correta sentada. Além disso, 90,9\% sabem da importância do uso de cremes hidratantes e $72,7 \%$ acreditam que o uso de almofadas com orifício no meio é um método de prevenção das LPP.

Tabela 4 - Conhecimento dos cuidadores sobre prevenção de LPP

\begin{tabular}{lll}
\hline \multicolumn{4}{c}{$\mathrm{n}$} & $\%$ \\
\hline Secar as zonas corporais minuciosamente ajuda a prevenir LPP? & 11 & 100 \\
\hline Verdadeiro & 0 & 0 \\
Falso & & \\
\hline Usar cremes hidratantes ajuda a prevenir LPP? & 10 & 90,9 \\
\hline Verdadeiro & 1 & 9,0 \\
Falso & & 72,7 \\
\hline Usar almofadas com orifício no meio ajuda a prevenir LPP? & 8 & 27,2 \\
\hline Verdadeiro & 3 & 100 \\
Falso & & 0 \\
\hline Tomar 1 litro de água por dia ajuda a prevenir LPP? & 11 & \\
\hline Verdadeiro & 0 & 100 \\
Falso & & 0 \\
\hline A correta postura sentada ajuda a prevenir LPP? & 11 & \\
\hline Verdadeiro & 0 & \\
Falso & 2019. & \\
\hline
\end{tabular}

Fonte: De Paula S, et al., 2019.

\section{DISCUSSÃO}

Pode-se identificar que o perfil dos cuidadores foi constituído, em sua maioria, por mulheres jovens, com relação de parentesco materno com a criança e nível fundamental de escolaridade, correspondendo aos achados encontrados em estudos similares (LUCENA NM, et al., 2016). O cuidador pode ser definido como a pessoa, membro ou não da família, que se responsabiliza por auxiliar na realização das tarefas diárias da pessoa com deficiência ou fragilidade física, o contexto da família, a mulher, geralmente, assume o papel de cuidadora principal de crianças com deficiências neuromotoras, dedicando-se integralmente ao seu cuidado. Diaz LJ, et al. (2017) salientam que a escolha da mulher como referência de suporte à criança está relacionada com o vínculo afetivo, a disponibilidade de tempo e o grau de parentesco, mesmo na carência de uma formação básica específica para a assistência.

Em relação às características do perfil clínico das crianças e adolescentes usuários de cadeiras de rodas, a pesquisa foi composta, principalmente, de participantes com diagnóstico de doenças neuromusculares. As doenças neuromusculares se constituem em um grupo de desordens, hereditárias ou adquiridas, que afetam não somente a unidade motora, mas também o trato cortiço espinhal, o cerebelo e as vias espinocerebelares. Além de complicações respiratórias e acentuados desvios posturais, esta população é considerada de risco para lesões crônicas na pele em virtude de significativos déficits no controle muscular, postural e pélvico na posição sentada (STROBL WM, 2013). Em um estudo recente realizado em 130 adultos com doenças neuromusculares, os autores encontraram uma prevalência de 14\% de LPP na amostra investigada. 
O estabelecimento precoce de estratégias de adequação postural em cadeira de rodas é essencial para melhorar a qualidade de vida de pacientes com deficiências neuromotoras e restrição de mobilidade (PELLEGRINI NA, 2016). As principais medidas de prevenção são a avaliação dos pacientes em risco; o manejo do estado nutricional incluindo a hidratação, a inspeção e a avaliação diária da pele; o manejo da umidade e a redistribuição da pressão através de mudanças de postura e manobras de alívio (SAMPAIO LR, et al., 2019).

A etiologia das lesões por pressão é complexa e multifatorial. No entanto, a sobrecarga tecidual tem sido definida como o fator determinante para o surgimento de feridas em pacientes acamados. A combinação de dano celular resultante da deformação tecidual prolongada, a isquemia dos tecidos moles e a interrupção do equilíbrio no sistema linfático são os principais mecanismos envolvidos no processo de surgimento de úlceras de pressão. Neste sentido, sabe-se que o efeito prejudicial da pressão sobre os tecidos está diretamente relacionado com a magnitude e a duração da pressão (SONENBLUM SE, SPRIGLE SH, MARTIN JS, 2016).

Apesar da evidente relação entre o tempo de permanência sentado e o surgimento de feridas na pele, 0 presente estudo mostrou que mais de $90 \%$ dos cuidadores não receberam orientações profissionais sobre medidas preventivas para LPP. Diversos estudos têm avaliado o nível de conhecimento e as atitudes de profissionais da saúde para a prevenção de LPP (MIYAZAKII MY, et al., 2010; PORTER-ARMSTRONG AP, et al., 2018; FRANÇA AP, et al., 2019).

A maioria das investigações demonstrou que estes profissionais apresentam déficits significativos sobre esta temática, apesar dos avanços técnico-científicos e dos protocolos internacionais de fácil acesso. Na esfera brasileira, estes resultados são ainda mais impactantes em virtude da escassez de guidelines clínicos nacionais para a prática baseada em evidências (MIYAZAKII MY, et al., 2010; FRANÇA AP, et al., 2019).

Em um recente estudo realizado no Canadá os autores observaram que $90 \%$ dos profissionais de saúde que responderam a um questionário estruturado estavam cientes da necessidade de implementar protocolos de prevenção de LPP. No entanto, $23 \%$ confirmaram que não há protocolos padronizados nas instituições que trabalham. Estes acham indicam que, mesmo em países desenvolvidos, há uma disparidade entre a necessidade de implementar protocolos baseados em evidências e os recursos disponíveis para sistematicamente monitorar e prevenir LPP. Dentre as barreiras que limitam a efetiva prevenção de LPP, incluem-se as limitações dos profissionais de saúde envolvidos no processo, incluindo as restrições técnicas, a inexperiência na área, sobrecarga de trabalho e falta de acesso a recursos estruturais (LEBLANC L, et al., 2019).

Considerando o tempo de permanência na cadeira de rodas, estudos em participantes adultos americanos mostraram uma média diária de 10-11 horas (SONENBLUM SE, et al., 2016; SONENBLUM SE, SPRIGLE $\mathrm{SH}, 2016)$. Estas investigações foram realizadas em pacientes lesados medulares que realizaram, em média, 16 transferências diárias. Em nosso estudo, os cuidadores referiram que as crianças e adolescentes permanecem mais de 13 horas por dia na posição sentada.

Estes resultados podem ser explicados pela dificuldade apresentada pelos participantes deste estudo de realizar as transferências de forma independente. Muitos fatores influenciam na habilidade de transferir-se da cadeira de rodas de forma independente, incluindo a idade do paciente, o tipo de cadeira de rodas, a presença de dor no ombro, a força muscular, o condicionamento físico e o treinamento de técnicas de transferências (KIRBY RL, et al., 2016).

Dentre os fatores extrínsecos, a pressão é considerada um dos principais aspectos fisiopatológicos envolvidos no aparecimento das LPP. Estudos tem estimado que, quando a pressão sobre os tecidos internos excede $32 \mathrm{mmHg}$ por um período superior a duas horas, inicia-se um processo de isquemia localizada que pode resultar em dano tecidual irreversível (BUCKI M, et al., 2012). Neste contexto, orientações sobre as manobras de alívio de pressão, tais como os push-ups e as inclinações laterais, são de fundamental importância, uma vez que essas abordagens restauram o fluxo sanguíneo e otimizam as necessidades metabólicas das células locais, reduzindo o risco de isquemias teciduais (VOS-DRAPER TL, MORROW MM, 2016). 
Na presente pesquisa, mais da metade dos participantes investigados afirmaram que não realizam as manobras de alívio. No estudo de Sonenblum SE e Sprigle SH (2016), os autores demonstraram uma correlação significativa entre o número de manobras de alívio desempenhadas ao longo do dia e o aparecimento de LPP, concluindo que quanto maior o número de manobras, menor a probabilidade de desenvolver feridas. Além disso, as investigações demonstram que as manobras de alívio, tais como as inclinações laterais para a distribuição do peso, podem reduzir $29-46 \%$ a pressão na região isquiática e aumentar em $100 \%$ o fluxo sanguíneo local (SONENBLUM SE, et al., 2014). No entanto, estudos controlados demonstraram que a duração mínima das manobras de alívio para a restauração do fluxo sanguíneo é de, aproximadamente, 60 segundos. Dos pacientes que afirmaram realizar as manobras ao longo do dia nesta investigação, $80 \%$ relataram que o tempo médio de alívio foi de 10 segundos, o que configura um tempo insuficiente para o restabelecimento do aporte sanguíneo adequado (COGGRAVE MJ, ROSE LS, 2003; COGAN AM, et al., 2017).

Apesar da carência de informações de profissionais da saúde e do escasso e inadequado uso das manobras de alívio, a maioria dos cuidadores demonstrou um moderado nível de conhecimento sobre medidas preventivas para as LPP. Os participantes do estudo relataram que aspectos como nutrição, postura e ingesta hídrica são fatores importantes no cuidado da pele dos pacientes com restrição de mobilidade. No entanto, é importante considerar que cuidados essenciais tais como a troca frequente de fraldas, a inspeção visual e a cautela com traumas acidentais foram pouco relatadas pelos entrevistados.

Sampaio LR, et al. (2019), em um estudo similar, observaram que, a maioria dos cuidadores de pessoas com mobilidade reduzida detinham apropriação dos conceitos chave pertinentes a etiologia das LPP. No entanto, ao serem abordados sobre as intervenções para prevenção, observou-se o desconhecimento das práticas preventivas. Os autores afirmam que o conhecimento apresentado sobre a enfermidade da pele não se traduziu satisfatoriamente em habilidades e atitudes para a prevenção. Corroborando nossos achados, os autores também afirmam que a carência de orientações por parte dos profissionais da saúde sobre as medidas eficazes de prevenção de LPP foram determinantes para os resultados encontrados.

Ressalta-se que mais de $80 \%$ das crianças e adolescentes participantes deste estudo não apresentaram histórico de LPP ao longo da vida. Segundo Artico, et al. (2018) a ausência de um cuidador treinado pode ser considerada um fator de risco para o desenvolvimento de feridas na pele. O National Pressure Ulcer Advisory Panel (NPUAP) afirmam que a educação de cuidadores (formais ou informais) de pessoas com mobilidade reduzida é uma estratégia altamente recomendada para o manejo, acompanhamento e prevenção das LPP (NPUAP, 2014). Estas evidências são sustentadas por outros protocolos internacionais que afirmam que estratégias educativas são requisitos fundamentais para a redução da prevalência de feridas cutâneas altamente incapacitantes. Dentre as temáticas sugeridas por estes guidelines estão: a informação sobre as causas das lesões, medidas preventivas, orientações sobre necessidades nutricionais e posicionamentos na cadeira de rodas e no leito (QASEEM A, et al, 2015).

Atualmente poucos estudos avaliam o conhecimento de cuidadores sobre a prevenção de LPP. Em um estudo polonês realizado com 62 cuidadores, os autores demonstraram que mais da metade da amostra investigada não recebeu nenhum tipo de informação sobre prevenção de LPP. Além disso, a maioria dos participantes desconhece medidas preventivas básicas (KWICZALA-SZYDLOWSKA et al., 2005). No Brasil, Silva ML, et al. (2009) realizaram um estudo qualitativo com sete cuidadores de pacientes portadores de lesões por pressão. O estudo revelou que a maioria dos cuidadores não recebeu orientações de profissionais da saúde.

Apesar disso, alguns entrevistados demonstraram possuir um conhecimento significativo sobre prevenção de lesões na pele, provavelmente com base no saber popular e empírico. Em relação ao uso de dispositivos para a prevenção de feridas, os protocolos internacionais não recomendam o uso de almofadas com orifícios no meio. As evidências demonstram que este recurso cria áreas de alta pressão que podem alterar a microcirculação e danificar os tecidos (NPUAP, 2014). Em nosso estudo, 72\% dos entrevistados, afirmaram que o uso de almofadas com orifício minimiza o aparecimento de feridas, reafirmando a necessidade do conhecimento técnico-científico para o desenvolvimento de programas educativos voltados para cuidadores.

REAS/EJCH | Vol.Sup.n.42 | e2437 | DOI: https://doi.org/10.25248/reas.e2437.2020 Página 8 de 10 


\section{CONCLUSÃO}

O presente estudo demonstrou que os cuidadores entrevistados apresentaram um moderado nível de conhecimento sobre a prevenção de lesões por pressão, provavelmente, com base no conhecimento popular e empírico. Em virtude disso, a pesquisa reforça a necessidade do desenvolvimento de programas educativos nacionais sobre medidas preventivas de lesões por pressão voltadas para os cuidadores e para os profissionais da saúde, reduzindo, desta forma, os custos ao sistema de saúde e melhorando a qualidade de vida de pacientes com mobilidade reduzida.

\section{REFERÊNCIAS}

1. ARTICO M, et al. Prevalence, incidence and associated factors of pressure ulcers in home palliative care patients: $A$ retrospective chart review. Palliat Med, 2018;32(1):299-307.

2. BELLON JA. Elaboración y validación de un instrumento para medir conocimientos de la persona cuidadora en prevención de úlceras por presión. Nure Investigacion, 2014;11(70):1-11.

3. BUCKI M, et al. Patient-specific finite element model of the buttocks for pressure ulcer prevention--linear versus nonlinear modelling. Comput Methods Biomech Biomed Engin, 2012;15 Suppl 1:38-40.

4. COGAN AM, et al. Systematic review of behavioral and educational interventions to prevent pressure ulcers in adults with spinal cord injury. Clin Rehabil, 2017;31(7):871-80.

5. COGGRAVE MJ, ROSE LS. A specialist seating assessment clinic: changing pressure relief practice. Spinal Cord, 2003;41(12):692-5.

6. DIAZ LJ, CRUZ DA. Modelo de adaptação em um ensaio clínico controlado com cuidadores familiares de pessoas com doenças crônicas. Texto contexto - enferm, 2017; 26(4): e0970017.

7. FRANÇA AP, et al. Conhecimento de enfermeiros sobre o manejo de lesões por pressão em unidade de terapia intensiva. Revista Eletrônica Acervo Saúde, 2019; 11(8).

8. KIRBY RL, et al. Manual Wheelchair Skills Training for Community-Dwelling Veterans with Spinal Cord Injury: A Randomized Controlled Trial. PLoS One, 2016;11(12): e0168330.

9. KWICZALA-SZYDLOWSKA S, et al. Pressure ulcer prevention--evaluation of awarness in families of patients at risk. Przegl Lek, 2005;62(12):1393-7.

10. LEBLANC K, et al. Professionals' Knowledge, Attitudes, and Practices Related to Pressure Injuries in Canada. Advances in Skin \& Wound Care, 2019; 32(5), 228-233.

11. LUCENA NMG, et al. Sociodemographic indicators and quality of life of caregivers of children with neuromotor changes. Man Ther, Posturology Rehabil J, 2016;14.

12. LUBOZ V, et al. Biomechanical modeling to prevent ischial pressure ulcers. J Biomech, 2014;47(10):2231-6.

13. MIYAZAKII MY, et al. Knowledge on Pressure Ulcer Prevention Among Nursing Professionals. Rev latinoam enferm, 2010;18(6).

14. NPUAD - NATIONAL PRESSURE ULCER ADVISORY PANEL, European Pressure Ulcer Advisory Panel. Prevention and Treatment of Pressure Ulcers: Quick Reference Guide. National Pressure Ulcer Advisory Panel. 2014.

15. OLIVEIRA DKS, et al. Perceptions of family caregivers and professionals in the family health strategy related to the care and neuropsychomotor development of children. Rev bras crescimento desenvolv hum, 2012;22(2):142-50.

16. PALFREYMAN SJ, STONE PW. A systematic review of economic evaluations assessing interventions aimed at preventing or treating pressure ulcers. Int J Nurs Stud, 2015;52(3):769-88.

17. PELLEGRINI N. Seated postural in wheelchair in NMD. Ann Phys Rehabil Med, 2016;59S:e82.

18. PORTER-ARMSTRONG AP, et al. Education of healthcare professionals for preventing pressure ulcers. Cochrane Database Syst Rev, 2018;5:CD011620.

19. QASEEM A, et al. Clinical Guidelines Committee of the American College of P. Risk assessment and prevention of pressure ulcers: a clinical practice guideline from the American College of Physicians. Ann Intern Med, 2015;162(5):359-69.

20. ROLIM JA, et al. Prevenção e tratamento de úlceras por pressão no cotidiano de enfermeiros intensivistas. Rev RENE, 2013;14:148-57.

21. SAMPAIO LR, et al. Prevenção de lesões por pressão: conhecimento e ações de cuidadores e pacientes domiciliares. $J$ Health NPEPS, 2019; 4(2):331-344.

22. SILVA ML, et al. Saberes e práticas de cuidadores domiciliares sobre úlcera por pressão: estudo qualitativo. Online braz j nurs, 2009;8(3).

23. SONENBLUM SE, et al. Everyday sitting behavior of full-time wheelchair users. J Rehabil Res Dev, 2016;53(5):58598. 
24. SONENBLUM SE, SPRIGLE SH. Some people move it, move it... for pressure injury prevention. J Spinal Cord Med, 2016:1-5.

25. SONENBLUM SE, et al. Effects of wheelchair cushions and pressure relief maneuvers on ischial interface pressure and blood flow in people with spinal cord injury. Arch Phys Med Rehabil, 2014;95(7):1350-7.

26. STROBL WM. Seating. J Child Orthop, 2013;7(5):395-9.

27. VOS-DRAPER TL, MORROW MB. Seating-Related Pressure Injury Prevention in Spinal Cord Injury: A Review of Compensatory Technologies to Improve In-Seat Movement Behavior. Curr Phys Med Rehabil Rep, 2016;4(4):320-8. 